\title{
Mesoscopic superpositions of states - approach to classicality and diagonalization in coherent state basis
}

\author{
G. S. Agarwal* \\ Physical Research Laboratory, Navrangpura, Ahmedabad-380009, India
}

\begin{abstract}
I consider the interaction of a superposition of mesoscopic coherent states and its approach to a mixed state as a result of a suitably controlled environment. I show how the presence of a gain medium in a cavity can lead to diagonalization in coherent state basis in contrast to the standard model of decoherence. I further show how the new model of decoherence can lead to the generation of $s$ ordered quasi distributions.
\end{abstract}

PACS Nos: 3.65.Bz, 42.50.Lc

Typeset using REVTEX

*also at Jawaharlal Nehru Centre for Advanced Scientific Research, Bangalore, India 
Mesoscopic superpositions of coherent states have been the subject of extensive studies [1] [7] because of their unusual interference characteristics and because of their relevance to the quantum measurement problem. These states are also known to be extremely sensitive to environmental interactions. The interference terms disappear fast and a kind of diagonalization takes place [1, 1 , 5]. The diagonalization is itself sensitive to the nature of the bath or the nature of the interaction with environment. If the initial state is a superposition of coherent states then ideally one would like to have a situation where the interaction with the bath produces a mixed state involving the two coherent states [6]. There are however difficulties as the bath itself has certain intrinsic properties [8] which must be satisfied and these intrinsic properties determine the dynamical characteristics of the subsystem. In this paper we examine the question - how a manipulation of the bath could possibly produce a diagonalization in coherent state basis.

We note that the subject of the manipulation of the bath has also attracted quite a bit of attention. Raimond et al. [5] demonstrated how the coupling of a high Q cavity containing the cat state to another resonator leads to the revival of coherence. Several authors [9] have shown how the feedback and other mechanisms could stabilize effects of decoherence. Poyatos et al. [10] demonstrated the engineering of the bath in the context of laser cooled trapped ions. There are other models of decoherence where the nonlinearities could give rise to coherence characteristics and could indeed produce new types of states [1]. Furthermore there exists the possibility [12 of achieving a control of the drift and diffusion terms in the dissipative dynamics by external electromagnetic field. The external fields make the environment nonthermal leading even to the possibility of making the drift term vanish and diffusion term rather small. There are several physical realizations of such pumped or nonthermal environment [13].

In this paper we consider the interaction of the field mode in a mesoscopic superposition state with a bath which consists of a gain medium in addition to the usual absorber. By choosing the gain appropriately we get purely diffusive motion of the field mode. This motion leads to diagonalization in coherent state basis though each coherent peak broadens 
due to diffusion. We also demonstrate how the time evolution under purely diffusive motion leads to the generation of the s-ordered quasi-distributions associated with the state of the field.

We start from a cat state, say, even or odd cat state for a bosonic system

$$
|\psi\rangle=\mathcal{N}_{ \pm}(|\beta\rangle \pm|-\beta\rangle)
$$

where the normalization constant is given by

$$
\mathcal{N}_{ \pm}^{-2} \equiv 2\left(1 \pm \exp \left(-2|\beta|^{2}\right)\right)
$$

The bosonic mode may, for example, represent a field mode in a cavity or the center of mass motion of an ion in a trap. The Wigner function $\Phi\left(\alpha, \alpha^{*}\right)$ for the state $(1)$ is

$$
\begin{aligned}
\Phi\left(\alpha, \alpha^{*}\right) & =\frac{2 N_{ \pm}^{2}}{\pi}\left(\exp \left\{-2|\alpha-\beta|^{2}\right\}+\exp \left\{-2|\alpha+\beta|^{2}\right\}\right. \\
& \left. \pm 2 \exp \left(-2|\alpha|^{2}\right) \cos (4 \beta y)\right) ; \quad \alpha=x+i y, \quad \beta=\text { real }
\end{aligned}
$$

The Wigner function thus consists of two Gaussians centered at $\alpha= \pm \beta$ with an interference term centered at the origin $\alpha=0$. The period of oscillation depends on $\beta$. The interaction with the environment is generally described by the density matrix equation [8] for the bosonic mode $a$

$$
\frac{\partial \rho}{\partial t}=-\kappa\left(a^{\dagger} a \rho-2 a \rho a^{\dagger}+\rho a^{\dagger} a\right)
$$

where $2 \kappa$ will be the rate of dissipation. The Wigner function at time $t$ will be given by

$$
\begin{aligned}
\Phi\left(\alpha, \alpha^{*}, t\right)= & \frac{2 \mathcal{N}_{ \pm}^{2}}{\pi}\left(\exp \left(-2\left|\alpha-\beta e^{-\kappa t}\right|^{2}\right)+\exp \left(-2\left|\alpha+\beta e^{-\kappa t}\right|^{2}\right)\right. \\
& \left. \pm 2 \exp \left(-2|\alpha|^{2}\right) \exp \left(-2 \beta^{2}\left(1-e^{-2 \kappa t}\right)\right) \cos \left(4 \beta y e^{-\kappa t}\right)\right) .
\end{aligned}
$$

We note that as a result of interaction with the environment the two Gaussians move towards each other eventually merging into one Gaussian. The amplitude of the oscillatory term goes down by a factor $\exp \left(-2 \beta^{2}\left(1-e^{-2 \kappa t}\right)\right)$ and the period of oscillation increases by $e^{\kappa t}$. For $\kappa t \gg 1$, Eq. (5) goes over to 


$$
\Phi \rightarrow \frac{2}{\pi} e^{-2|\alpha|^{2}}
$$

For completeness we show this evolution in Fig.1 for different values of $\kappa t$.

Intuitively, the emergence of classical behavior [6, 14] on interaction with the environment would require a different behavior - we would expect to see a double Gaussian structure with the missing oscillatory behavior. A natural question arises what model of environment could achieve that. One natural possibility is to consider a situation so that the exponentially damped factors can be removed. For example, one could think of inserting a gain media in the context of cavity problems. The gain can be chosen so as to compensate the loss. Thus one might be able to keep the double Gaussian structure. However, any gain also introduces some noise. We thus examine in detail the consequences of both gain and loss on the dynamics of a mesoscopic superimposition of states. Let $2 \Gamma$ be the gain of the gain medium. Then Eq.(3) is modified to

$$
\dot{\rho}=-\kappa\left(a^{\dagger} a \rho-2 a \rho a^{\dagger}+\rho a^{\dagger} a\right)-\Gamma\left(a a^{\dagger} \rho-2 a^{\dagger} \rho a+\rho a a^{\dagger}\right) .
$$

The Wigner function obeys the equation of motion

$$
\frac{\partial \Phi}{\partial t}=(\kappa-\Gamma) \frac{\partial}{\partial \alpha}(\alpha \Phi)+\frac{\kappa+\Gamma}{2} \frac{\partial^{2} \Phi}{\partial \alpha \partial \alpha^{*}}+c . c . .
$$

On writing $\alpha=x+i y$, we get

$$
\frac{\partial \Phi}{\partial t}=(\kappa-\Gamma) \frac{\partial}{\partial x}(x \Phi)+(\kappa-\Gamma) \frac{\partial}{\partial y}(y \Phi)+\left(\frac{\kappa+\Gamma}{4}\right)\left(\frac{\partial^{2}}{\partial x^{2}}+\frac{\partial^{2}}{\partial y^{2}}\right) \Phi .
$$

We now have two parameters $\kappa$ and $\Gamma$ which could be manipulated independently to produce the desired result.

Now the drift and diffusion coefficients are respectively equal to $(\kappa-\Gamma)$ and $(\kappa+\Gamma) / 4$. We now have the possibility of making drift vanish by choosing $\kappa=\Gamma$ leading to

$$
\frac{\partial \Phi}{\partial t}=2 \kappa \frac{\partial^{2} \Phi}{\partial \alpha \partial \alpha^{*}}
$$

The general solution of (10) can be expressed as 


$$
\Phi\left(\alpha, \alpha^{*}, t\right) \equiv \frac{1}{\pi \delta} \int \exp \left(-\left|\alpha-\alpha_{0}\right|^{2} / \delta\right) \Phi\left(\alpha_{0}, \alpha_{0}^{*}, 0\right) d^{2} \alpha_{0} ; \quad \delta=2 \kappa t
$$

On substituting (3) in (11) and on using the identity

$$
\int \frac{d^{2} z}{\pi} \exp \left(\alpha z+\beta z^{*}-\gamma|z|^{2}\right)=\frac{1}{\gamma} \exp \left(\frac{\alpha \beta}{\gamma}\right),
$$

we get

$$
\begin{aligned}
\Phi\left(\alpha, \alpha^{*}, t\right)= & \frac{2 \mathcal{N}^{2}}{\pi(1+2 \delta)}\left(\exp \left\{-\frac{2}{(2 \delta+1)}|\alpha-\beta|^{2}\right\}+\exp \left\{-\frac{2}{(2 \delta+1)}|\alpha+\beta|^{2}\right\}\right. \\
& \left. \pm 2 \exp \left\{-\frac{2|\alpha|^{2}}{(1+2 \delta)}-\frac{4 \beta^{2} \delta}{1+2 \delta}\right\} \cos \left(\frac{4 \beta y}{1+2 \delta}\right)\right) .
\end{aligned}
$$

This result should be compared with the standard model Eq.(5) of decoherence.

Thus for the interaction of a field mode in a Cat state with the new environmental conditions, each component in the Wigner function remains located at the original position as there is no drift in the model. However, each component undergoes diffusion. For the usual model of decoherence there is no diffusion although the mean position quickly drifts towards origin. The period of oscillation of the interference term increases. The amplitude of oscillation also decreases. For larger $\delta$ and for $\beta^{2}>\delta+\frac{1}{2}$, the oscillatory (interference) term disappears leading to

$$
\Phi\left(\alpha, \alpha^{*}, t\right) \approx \frac{2 \mathcal{N}_{ \pm}^{2}}{\pi(1+2 \delta)}\left(\exp \left(-\frac{2}{(2 \delta+1)}|\alpha-\beta|^{2}\right)+\beta \rightarrow-\beta\right) .
$$

We thus achieve diagonalization in coherent state basis - the decoherence to a mixed state which is a superposition of two Gaussians at $\pm \beta$. This is what we had set out to achieve. We show in Fig. 1 the effects of decoherence on the Wigner function of the field mode interacting with this new model of the environment. These results should be compared with the ones for the standard model of decoherence. There are obviously important differences in the dynamics of a Cat state interacting with different types of environment.

We next present some very general results on various quasi distributions like the Pfunction, the Q-function and the Wigner function. We discuss the parameter regime in which the nonclassical characteristics of the original state start disappearing. For this purpose we 
examine the equation of motion for the characteristic function $\left\langle\exp \left(\gamma a^{\dagger}-\gamma^{*} a\right)\right\rangle$ which is the Fourier transform of $\Phi$. Clearly the characteristic function obeys the equation

$$
\frac{\partial}{\partial t}\left\langle\exp \left(\gamma a^{\dagger}-\gamma^{*} a\right)\right\rangle=-2 \kappa|\gamma|^{2}\left\langle\exp \left(\gamma a^{\dagger}-\gamma^{*} a\right)\right\rangle
$$

and hence

$$
\left\langle\exp \left(\gamma a^{\dagger}(t)-\gamma^{*} a(t)\right)\right\rangle=\exp \left(-2 \kappa|\gamma|^{2} t\right)\left\langle\exp \left(\gamma a^{\dagger}-\gamma^{*} a\right)\right\rangle
$$

which on using the disentangling theorem leads to

$$
\left\langle\exp \left(\gamma a^{\dagger}(t)\right) \exp \left(-\gamma^{*} a(t)\right)\right\rangle \equiv \exp \left(-\left(2 \kappa t-\frac{1}{2}\right)|\gamma|^{2}\right)\left\langle\exp \left(\gamma a^{\dagger}-\gamma^{*} a\right)\right\rangle
$$

Note that the Fourier transform of the left hand side yields the quasi distribution known as the P-function of the system. Thus from (17) we conclude that the P-function at time such that $2 \kappa t=\frac{1}{2}$ is equal to the Wigner function at $t=0$ and the P-function at time given by $2 \kappa t=1$ is equal to the Q-function at $t=0$. This implies that all nonclassical effects [15] will disappear at times given by $2 \kappa t \geq 1$. Furthermore, the $P$-function definitely exists as an ordinary function in the interval $1 \geq 2 \kappa t \geq \frac{1}{2}$ though it can be negative. Eq.(16) also shows that the Wigner function at time $t$ is equal to the s-parameterized distribution [16] $\Phi$ s at time $t=0$. This is because the s-parameterized distribution is the Fourier transform of $\exp \left[s|\gamma|^{2} / 2\right]\left\langle\exp \left(\gamma a^{\dagger}-\gamma^{*} a\right)\right\rangle$. Clearly, for our problem, $s$ is equal to $-4 \kappa t$. Note that for $s=-1$, we get the Q-function, i.e. the Wigner function at time $2 \kappa t=\frac{1}{2}$ is equal to the Q-function at $t=0$.

We note in passing that if $\Gamma$ is related to $\kappa$ via the relation

$$
\frac{\Gamma}{\kappa}=\frac{\bar{n}}{(\bar{n}+1)} \leq 1
$$

then the model (7) describes the interaction with a thermal bath 17, 18. However, $\Gamma$ could exceed $\kappa$ as we are describing a pumped environment. We could thus refer to the model (7) without the condition (18) as the nonthermal and phase sensitive environment. 
In summary, we have shown how the introduction of a gain medium can produce very remarkable modifications in the dissipative dynamics of a superposition of mesoscopic states. We demonstrated how to achieve classicality and diagonalization in coherent state basis.

The author thanks R.P. Singh, S. Menon for the beautiful graphics and J. Kupsch, W. Schleich for discussions on decoherence. 


\section{REFERENCES}

[1] V. Buzek and P. L. Knight, in "Progress in Optics", ed. E. Wolf (North Holland, Amsterdam, 1995) vol. 34, p. l; S. J. D. Phoenix, Phys. Rev. A 41, 5132 (1990); L. Davidovich, M. Brune, J. M. Raimond and S. Haroche, Phys. Rev. A 53, 1296 (1996); V. Buzek, A. Vidiella - Barranco and P. L. Knight, Phys. Rev. A 45, 6570 (1992).

[2] C. Monroe, D. M. Meekhof, B. E. Knig and D. J. Wineland, Science 272, 1131 (1996); D. Leibfried, D. M. Meekhof, B. E. Knig, C. Monroe, W. M. Itano and D. J. Wineland, Phys. Rev. Lett. 77, 4281 (1996).

[3] R. L. deMatos Filho and W. Vogel, Phys. Rev. Lett. 76, 608 (1996).

[4] M. Brune, E. Hagley, J. Dreyer, X. Maitre, A. Maali, C. Wunderlich, J. M. Raimond and S. Haroche, Phys. Rev. Lett. 77, 4887 (1996).

[5] J.M. Raimond, M. Brune and S. Haroche Phys. Rev. Lett. 79, 1964 (1997); S. Haroche, Physics Today, July 1998, p.36.

[6] E. Joos in D. Giulini et al., "Decoherence and the Appearance of a classical world in Quantum Theory" (Springer Berlin, 1996) p.35; W.H. Zurek, Prog. Theoret. Phys. 89, $281(1993)$.

[7] J. J. Slosser and G. J. Milburn, Phys. Rev. Lett. 75, 418 (1995); C.C. Gerry, Opt.Commun. 91, 247 (1992).

[8] See for example H. Haken, Laser Theory in "Handbuch der Physik", Ed. S. Flugge, (Springer, Berlin 1970) Vol.XXV/2C, p.56; C.W. Gardiner, "Handbook of Stochastic Processes" (Springer, Berlin 1983) Chap. X.

[9] D. Vitali, P. Tombesi and G.J. Milburn, Phys. Rev. Lett. 79, 2442 (1997); J. Mod. Optics, 44, 2033 (1997); D. B.Horoshko and S. Ya. Kilin, Phys. Rev. Lett. 78, 840(1997); J. Mod. Optics 44, 2043 (1997); L. Viola and S. Lloyd, Phys. Rev. A (1998), in press; G. Vemuri, K. V. Vasavada and G. S. Agarwal, Phys. Rev. A50, 2599 (1994). 
[10] J. F. Poyatos, J. I. Cirac and P. Zoller, Phys. Rev. Lett. 77, 4738 (1996).

[11] G. S. Agarwal, J. Opt. Soc. Am. B 5, 1940 (1988); H. Ezaki, G. S. Agarwal and E. Hanamura, Opt. Commun. 138, 65 (1997); G. C. Gerry and E. E. Hach, Phys. Lett. A 174, 185 (1993).

[12] G. S. Agarwal, Phys. Rev. A 34, 4055 (1986); S. Stenholm, D. A. Holm and M. Sargent, Phys. Rev. A 31, 3124 (1985).

[13] G. S. Agarwal, Phys. Rev. A 54, R3734 (1996); A. L.Gaeta, R. W. Boyd and G. S. Agarwal, Phys. Rev. A 46, 4271 (1992); S. T. Ho, P. Kumar and J. H. Shapiro, J. Opt. Soc. Am B 8, 37 (1991); A. Rosenhouse-Dantsker, A. D. Wilson-Gordon and H. Friedmann, Phys. Rev. A 52, 4839 (1995).

[14] Several recent publications discuss the question of approach to classical behavior of a quantum system when interactions with the bath are included. S. Habib, K. Shizume and W. H. Zurek, Phys. Rev. Lett. 80, 4361 (1998); B. G. Klappauf, W. U. Oskay, D. A. Steck and M. G. Raizen, Phys. Rev. Lett. 81, 1203 (1998).

[15] Transformation of nonclassical states by a phase insensitive amplifier was studied in detail: C. K. Hong, S. Friberg and L. Mandel, J. Opt. Soc. Am B 2, 494 (1985); G. S. Agarwal and K. Tara, Phys. Rev. A 47, 3160 (1993).

[16] See, for example, U. Leonhardt, "Measuring the Quantum State of Light" (Cambridge University Press 1997) Eq.(3.60).

[17] The model (7) with the condition (18) is a special case of a more general model [G. S. Agarwal, Phys. Rev. A 4, 739 (1971)], which was used by G. J. Milburn and D. F. Walls,Phys. Rev. A 38, 1087 (1988) for studying decoherence.

[18] Effects of thermal environment on the Cat states are reported in M.S. Kim and V. Buzek, J. Mod. Opt. 39, 1609 (1992). 


\section{FIGURES}

FIG. 1. Diagonalization in coherent state basis of a Cat State $(|\beta\rangle+|-\beta\rangle)$. These frames show the behavior of the Wigner function as a function of $\alpha=x+i y$. The plots on the left show the results [Eq.(13)] for the new model of decoherence due to a controlled environment consisting of a gain medium, whereas the plots on right show the results [Eq.(5)] for the standard model of decoherence. The z-axis gives the numerical values of the Wigner function. The plot a0 gives the Wigner function at time $\mathrm{t}=0$. The subsequent plots are for increasing times for $\delta=2 \kappa t=0.1$ for $\mathrm{a} 1, \mathrm{~b} 1 ; 0.5$ for $\mathrm{a} 2, \mathrm{~b} 2 ; 4.0$ for a3, b3. We have set $\beta=3$ for all the plots. 


$$
\Delta
$$


22

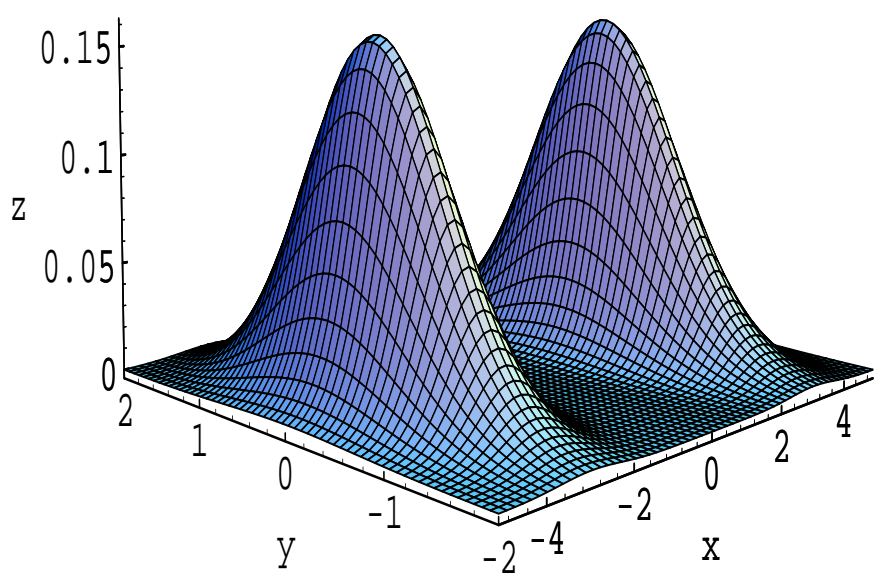

a3

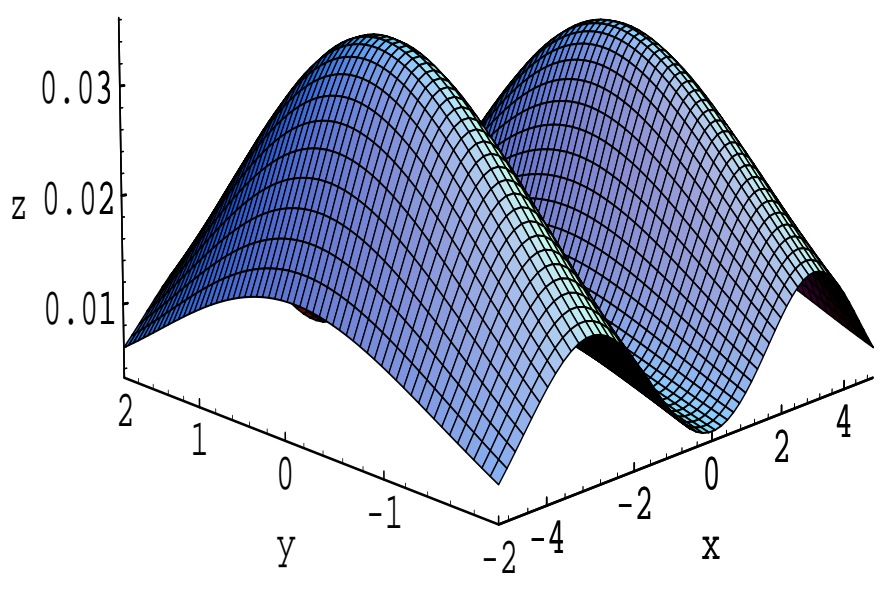

b2

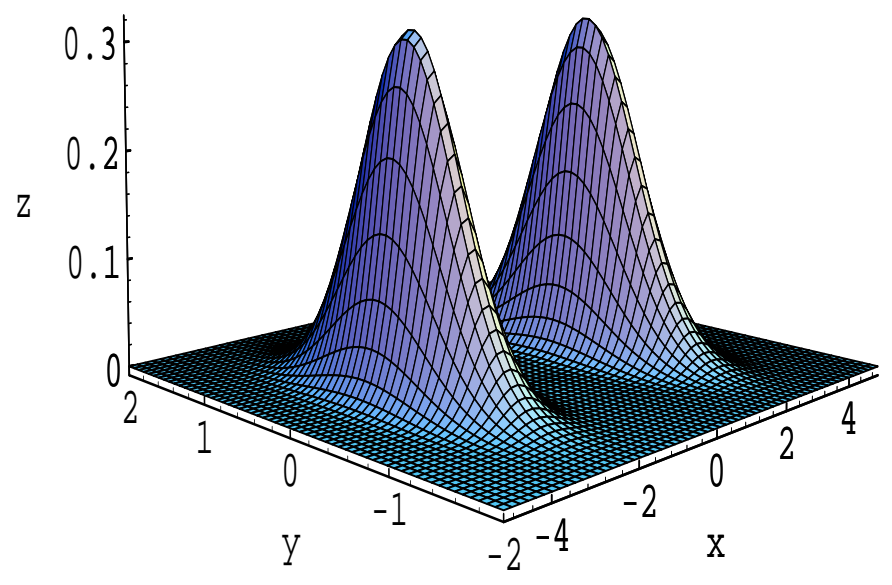

b3

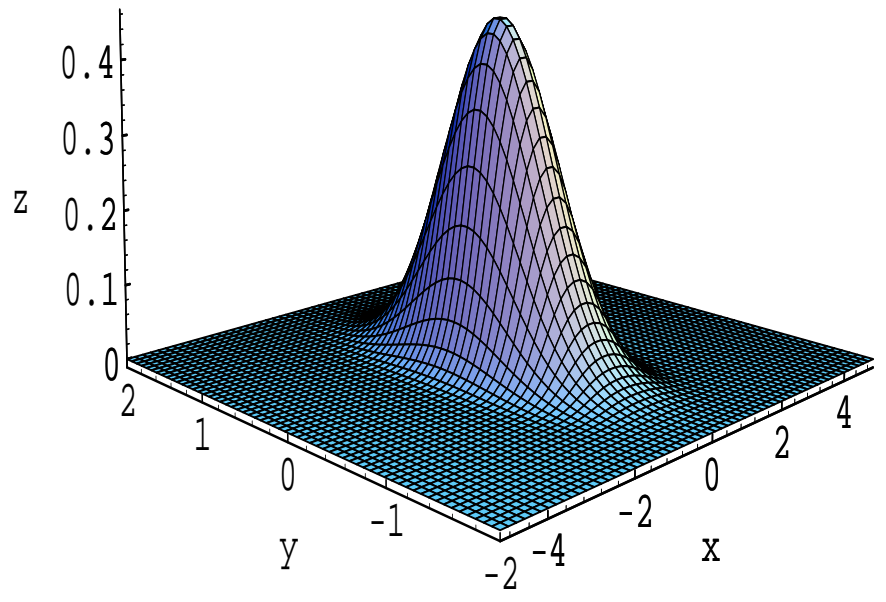

